\title{
Coupled nuclear-electronic dynamics in photoionization of $\mathrm{H}_{2}$
}

\author{
Laura Cattaneo ${ }^{1 *}$, Jannie Vos ${ }^{1}$, Roger Yulio Bello ${ }^{2}$, Alicia Palacios ${ }^{2}$, Sebastian Heuser ${ }^{1}$, \\ Luca Pedrelli ${ }^{1}$, Matteo Lucchini ${ }^{1}$, Claudio Cirelli ${ }^{1,3}$, Fernando Martín ${ }^{2}$ and Ursula Keller ${ }^{1}$ \\ ${ }^{1}$ Physics Department, ETH Zurich, 8093 Zurich, Switzerland \\ ${ }^{2}$ Departamento de Química, Módulo 13, Universidad Autónoma de Madrid, 28049 Madrid, Spain \\ ${ }^{3}$ Empa - Swiss Federal Laboratories for Materials Science \& Technology, 8600 Dübendorf, \\ Switzerland
}

\begin{abstract}
In this study we investigate the dissociative photoionization of molecular hydrogen $\mathrm{H}_{2}$, addressing the influence of autoionizing states and nuclear motion on the photoelectron dynamics. Experimental results are compared with ab initio calculations.
\end{abstract}

\section{Introduction}

Attosecond science is recently showing an increasing interest in the real-time investigation of dynamical processes in molecules [1-4]. In particular molecular photoionization is a much richer phenomenon when compared to the atomic case, with a strong spatial dependence due to the complex nature of the molecular scattering potential [4] and the still debated coupling between the nuclear and electron degree of freedom. So far, the few existing attosecond experiments performed on molecules have allowed one to study electron dynamics decoupled from nuclear dynamics by assuming that the nuclei move much slower than electrons [2,3]. Therefore the former can be considered as "frozen in their original positions" while the fast electron dynamics evolve. However, even in the attosecond time regime, this approximation is usually inappropriate. In molecules composed of light atomic species like hydrogen, the nuclear motion can be as fast as electron dynamics, resulting in a strong coupling between them [5-8]. Here we address the question of what happens when nuclear motion occurs on the same time scale as electronic motion by performing state-of-the-art experiments and theoretical calculations.

To perform a benchmark-type of experiment, we have chosen the lightest and smallest of all molecules, $\mathrm{H}_{2}$. We benefit from a full coincidence detection scheme using a Cold Target Recoil Ion Momentum Spectroscopy (COLTRIMS) apparatus [9], implemented here to perform attosecond measurements. In addition we applied a nearly exact ab initio theory to describe both electronic and nuclear motions, as well the coupling between them, thus providing a stronger support to our conclusions [10].

For the first time, we unambiguously show that ionization delays can significantly depend on both photoelectron kinetic energy and the nuclear kinetic energy, which obliges us to extend the concept of ionization delays introduced for atomic systems. Variations of

* Corresponding author: claura@phys.ethz.ch 
ionization delays with the nuclear kinetic energy can be as large as variations with the electron kinetic energy, which implies that whenever light atoms are involved in the molecular ionization process, the outgoing electron wave packet cannot be disentangled from the nuclear wave packet.

The impact of this work goes well beyond the simple $\mathrm{H}_{2}$ molecule because $\mathrm{H}$ atoms are present in most organic and biologically relevant molecules. Thus, understanding the effects and contributions from coupled electron and nuclear dynamics present in such systems is of fundamental importance in many fields of research. In this study we investigate the dissociative photoionization (DPI) dynamics of $\mathrm{H}_{2}$ in the energy region where direct paths, involving the two lowest electronic states, ${ }^{2} \Sigma_{\mathrm{g}}{ }^{+}\left(1 \mathrm{~s} \sigma_{\mathrm{g}}\right)$ and ${ }^{2} \Sigma_{\mathrm{u}}{ }^{+}\left(2 \mathrm{p} \sigma_{\mathrm{u}}\right)$ (represented in Fig. 1b as solid black lines) of the molecular ion, can directly compete with autoionizing processes upon population of doubly excited states (DES, represented in Fig. $1 \mathrm{~b}$ as green-dash and red-dot lines) [11-13].

\section{$2 \mathrm{H}_{2}$ dissociative pathway selection and observations [10]}

$\mathrm{H}_{2}$ DPI can evolve either (i) along the two lowest electronic states, ${ }^{2} \Sigma_{\mathrm{g}}{ }^{+}\left(1 \mathrm{~s} \sigma_{\mathrm{g}}\right)$ and ${ }^{2} \Sigma_{\mathrm{u}}{ }^{+}\left(2 \mathrm{p} \sigma_{\mathrm{u}}\right)$ or (ii) via the excitation of the $\mathrm{Q}_{1}$ and $\mathrm{Q}_{2}$ series of the DES and subsequent autoionization. These paths evolve towards the same dissociation limit of the molecule at $18.08 \mathrm{eV}$ (see Fig. 1b). Using a reaction microscope detector we can at first identify and select the reaction pathways leading to the dissociation of the $\mathrm{H}_{2}$ molecule, in accordance with the $\mathrm{H}^{+}$-ion time of flight. Moreover, thanks to the 3D momenta coincidence detection of all charged particles ( $\mathrm{H}^{+}$and e-) we can reconstruct the kinematics of the neutral $\mathrm{H}$ particle following the total momentum conservation and thus retrieve the molecular frame before dissociation, i.e. the $\beta$ angle as represented in the inset of Fig. 1b. This allows us to select different molecular orientations with respect to the XUV polarization axis and thereby select the final state of the photoemitted electrons. In particular $\mathrm{Q}_{1}$ and $\mathrm{Q}_{2} \mathrm{DES}$ can be populated by molecules preferentially aligned parallel and perpendicular to the XUV polarization axis, respectively.

Thanks to the reconstruction of the missing neutral H-particle, we can access the kinetic energy of the $\mathrm{H}+\mathrm{H}^{+}$nuclear fragments, also known as Kinetic Energy Release (KER), a fundamental parameter employed to disentangle almost exclusively direct from autoionizing paths [14].

Combining both the angular- and KER-resolution we can select, for each RABBITT trace, electrons photoemitted from $\mathrm{H}_{2}$ molecules oriented within a defined angle with respect to the XUV polarization axis and with a defined $\left(\mathrm{KER}+\mathrm{K}_{\mathrm{e}}\right.$, where $\mathrm{K}_{\mathrm{e}}$ is the electron kinetic energy) energy. This consequently allows for the extraction of KER and $\beta$-angle resolved RABBITT phases as shown in Fig 1c and 1d.

In particular in both orientations, perpendicular (Fig. 1c) and parallel (Fig. 1d), we observe a clear modulation of the extracted phases not only where the DES induce explicit jumps in the molecular phase, but also where a zero-phase variation is expected. This non-zero phase modulation is attributed to the nuclear-electronic coupling degree of freedom.

The experimental observations in the parallel orientation have been compared with calculations performed by solving the time-dependent Schrödinger equation employing a basis of states that allows us to accurately describe molecular autoionization and the electron-nuclear couplings (see inset in Fig. 1d).

We found an overall very good agreement between theory and experiment, supporting the interpretation that the phase of the escaping electron wavepacket is a sensitive probe not only of the electron dynamics but also of the nuclear dynamics triggered in the photoionization process of $\mathrm{H}_{2}$, thus requiring a re-examination of its definition. 

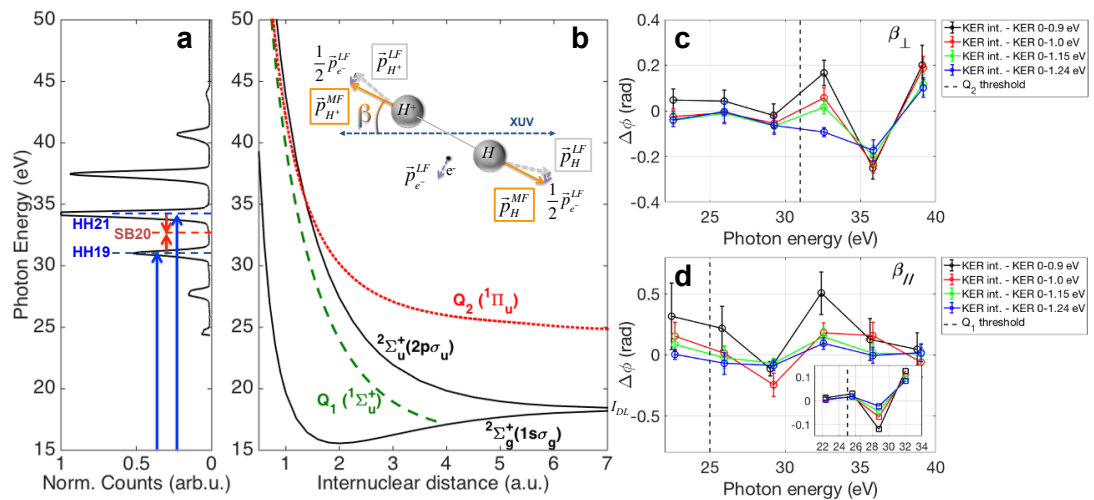

Fig. $1 \mathrm{H}_{2}$ dissociative photoionization. a) Example of an XUV spectrum used to perform the experiments in $\mathrm{H}_{2}$. b) Representation of the relevant potential energy curves as a function of the internuclear distance. For photon energies above $25 \mathrm{eV}$ the $\mathrm{Q}_{1}{ }^{1} \Sigma_{\mathrm{u}}{ }^{+}$state is dominantly populated, while the $\mathrm{Q}_{2}{ }^{1} \Pi_{\mathrm{u}}$ appears only for absorbed energies above $32 \mathrm{eV}$. On the top right a schematic representation of the ion momenta is reported, passing from the laboratory frame (LF, grey dashed arrows) to the molecular frame (MF, orange solid arrows) by summing half of the electron momentum (black dashed arrow). The resulting molecular orientation (solid grey line) with respect to the XUV polarization axis (blue dashed line) defines the $\beta$ angle. c) and d) represent the relative molecular phases as a function of the photon energy corresponding to $b_{\perp}$ and $b_{/ /}$, respectively. The black dashed lines indicate the $\mathrm{Q}_{2} \mathrm{c}$ ), and $\mathrm{Q}_{1}$ d) DES thresholds. Each experimental data point represents the weighted mean over a set of 8 independent measurements. The inset in the panel d) shows the ab initio calculations accounting for all interaction terms reproducing the experimental parallel case.

\section{References}

1. G. Sansone, et al., Nature 465, 763-766 (2010).

2. S. Haessler, et al., Phys. Rev. A 80, 011404 (2009).

3. M. Huppert, et al., Phys. Rev. Lett. 117, 093001 (2016).

4. P. Hockett, et al., J. Phys. B: At. Mol. Opt. 49, 095602 (2016).

5. A. D. Bandrauk, S. Chelkowski, H. S. Nguyen, Int. J. Quant. Chem. 100, 834-844 (2004).

6. C. R.Calvert, et al., Phys. Rep. 491, 1-28 (2010).

7. M. Lara-Astiaso, et al., Faraday Discuss 194, 41-59 (2016).

8. M. Vacher, et al., Phys. Rev. Lett. 118, 083001 (2017).

9. M. Sabbar, et al. , Rev. Sci. Instrum. 85, 103113 (2014).

10. L. Cattaneo, et al., Nature Phys., 14, 733-738 (2018).

11. M. Wickenhauser, et al., Phys. Rev. Lett. 94, 023002 (2005).

12. J. L. Sanz-Vicario, H. Bachau, F. Martin, Phys. Rev. A 73, 033410 (2006).

13. M. F. Kling, et al., Science 312, 246-248 (2006).

14. I. Sánchez, F. Martín, Phys. Rev. A 57, 1006 (1998). 